\title{
Crystal Structure of the 1:1 Complex between N,N,3,5- Tetramethylaniline and Hexafluorobenzene. Molecular-Packing Analysis of Complexes between Aromatic Amines and Hexafluorobenzene
}

\author{
Tor Dahl
}

Institute of Mathematical and Physical Sciences, University of Tromsø, P.O. Box 953, N-9001 Tromsø, Norway

\begin{abstract}
Dahl, T., 1989. Crystal Structure of the 1:1 Complex between $N, N, 3,5$-Tetramethylaniline and Hexafluorobenzene. Molecular-Packing Analysis of Complexes between Aromatic Amines and Hexafluorobenzene. - Acta Chem. Scand. 43: 172-176.

The title compound crystallizes in the monoclinic space group $P{ }_{1} / m$ with cell dimensions $a=7.038(2), b=13.904(4), c=8.504(4) \AA, \beta=102.51(3)^{\circ}$. The structure was refined by constraints to $R=0.058$ for 841 observed reflections. The structure is disordered with the hexafluorobenzene molcule in two equivalent orientations. The molecules are stacked alternately with a mean interplanar distance of $3.51 \AA$.

A molecular-packing analysis was performed for the title compound and for the complexes of hexafluorobenzene with $N, N, N^{\prime}, N^{\prime}$-tetramethyl-p-phenylenediamine and $N, N$-dimethylaniline. The analysis was based on lattice-energy calculations using atom-to-atom potential functions. The molecular packing giving the lowest calculated energy is very similar to that of the experimental structure for the complex with $N, N$-dimethylaniline, but more different for the two other complexes.
\end{abstract}

Crystal structures of molecular complexes of hexafluorobenzene (HFB) with aromatic hydrocarbons and aromatic amines show a relatively coherent pattern regarding molecular overlap and interplanar distance in the stack. ${ }^{1}$ The molecular overlap in the complex with $N, N, N^{\prime}, N^{\prime}$-tetramethyl-p-phenylenediamine (TMPDA) does not, however, conform to this pattern. ${ }^{1,2}$ Charge-transfer bands have been observed in the spectra of complexes between HFB and aromatic amines. ${ }^{3}$ As TMPDA is known as a very strong electron donor, the unusual molecular overlap in the complex with HFB may possibly be an effect of the chargetransfer interaction. The ionization potential of $N, N, 3,5-$ tetramethylaniline (TMA) is higher than that of TMPDA, but indicates that TMA is also a relatively strong electron donor. ${ }^{4,5}$ The purpose of investigating the complex of HFB with TMA was to see if this structure shows deviations from the pattern similar to those observed for the complex with TMPDA.

In order to discuss the possible effects on the structures of special kinds of intermolecular interactions in these complexes it is important to know the effects of the more traditional kinds of interactions. Lattice-energy calculations based on atom-to-atom potential functions were therefore performed for the two complexes mentioned and for the complex between $N, N$-dimethylaniline (DMA) and HFB. For this complex a relatively accurate low-temperature structure determination has been performed. ${ }^{6}$ The structure of the complex between HFB and $N, N$-dimethyl$p$-toluidine at room temperature has also been investigated, ${ }^{7}$ but the details of this disordered structure are re- garded as uncertain. This complex was therefore not included in the packing analysis.

\section{Experimental}

Colourless crystals of the title compound were obtained by evaporation at $-20^{\circ} \mathrm{C}$ of a solution of the two components in dichloromethane. The crystals are unstable on being exposed to the atmosphere and were kept in sealed capillaries. They are damaged by even moderate cooling and the data collection had therefore to be done at room temperature. Many of the crystals were twins with (001) as the twin plane. The one used for data collection was a single crystal with the approximate dimensions $0.5 \times 0.1 \times 0.3 \mathrm{~mm}$, along the $a$-, $b$ - and $c$-axis, respectively.

The cell parameters and X-ray intensities were measured on an Enraf-Nonius CAD4 diffractometer using $\mathrm{CuK \alpha}$ radiation $(\lambda=1.5418 \AA)$. The cell parameters were determined from the setting angles of 25 reflections. The intensities were collected for reflections with $\theta<70^{\circ}$ by an $\omega / 2 \theta$ scan at a rate in $\omega$ of $0.3-1.3^{\circ} \mathrm{min}^{-1}$. For the structure determination the 841 reflections with $I>1.5 \sigma(I)$ were used. Corrections were made for a continuous reduction down to $96 \%$ of the original intensities of the standard reflections, for Lorentz and polarization effects, and for absorption using an empirical method. ${ }^{8}$

Crystal data. $N, N, 3,5$-Tetramethylaniline hexafluorobenzene, $\mathrm{C}_{10} \mathrm{H}_{15} \mathrm{~N} \cdot \mathrm{C}_{6} \mathrm{~F}_{6}, M_{\mathrm{r}}=335.29$, space group $P 2_{1} / m$ (No. 11), $a=7.038(2), b=13.904(4), c=8.504(4) \AA, \beta=$ 
102.51(3) ${ }^{\circ}, V=812.4(5) \AA^{3}, Z=2, D_{\mathrm{x}}=1.370 \mathrm{~g} \mathrm{~cm}^{-3}$, $\mu\left(\mathrm{Cu} K_{\alpha}\right)=11.72 \mathrm{~cm}^{-1}$.

Structure determination and refinement. The positions of all non-H atoms of the TMA molecule and the approximate location of the HFB molecule were found from a Patterson map and successive Fourier syntheses. In order to obtain a satisfactory $R$-value, disorder had to be introduced with two orientations of the HFB molecule. A model for the disorder with two non-equivalent orientations, each with a mirror plane of the HFB molecule in the crystallographic mirror plane (010) turned out to be wrong. Satisfactory results were obtained using a model with two equivalent orientations as shown in Fig. 1. All $\mathrm{H}$ atoms could be located from a difference map.

In the last part of the least-squares refinement positional parameters for all atoms and thermal parameters, anisotropic for non- $\mathrm{H}$ atoms and isotropic for $\mathrm{H}$ atoms, were varied. Exceptions were made for parameters fixed by the symmetry and for the $U_{22}$ parameters of $\mathrm{F}(1), \mathrm{C}(1)$ and $\mathrm{C}(4)$, which are situated very close to the mirror plane (010). These $U_{22}$ parameters were given the values 0.35 , 0.15 and $0.15 \AA^{2}$, respectively, and kept constant in the refinement. Secondary extinction turned out to be important, and an isotropic extinction parameter was therefore refined. ${ }^{9}$

Because of the disorder and the large thermal vibrations of the HFB molecule, constrained refinement was used. ${ }^{10}$ The constraints were treated as extra observations and added to the structure factors in the least-squares refinement. They were given standard deviations of $0.005-0.010$ $\AA$ for atomic positions, $0.5-1.0^{\circ}$ for bond angles and 0.02 $0.03 \AA^{2}$ for $U$-values. All C-H distances were constrained to $1.00 \AA$ and the angles $\mathrm{C}\left(\right.$ or N)$-\mathrm{C}($ methyl $)-\mathrm{H}$ to $109^{\circ}$. All further constraints refer to the HFB molecule. The molecule was constrained to be planar and all bond angles to $120^{\circ}$. The bond lengths were constrained to the same value for all $\mathrm{C}-\mathrm{F}$ bonds and for all $\mathrm{C}-\mathrm{C} /$ bonds. The sums of the $U_{11}$ values were constrained such that they were equal for the atoms on each side of $(010)$. For a large number of pairs of atoms in the molecule the thermal vibrations along the interatomic vectors were constrained such that they were equal.

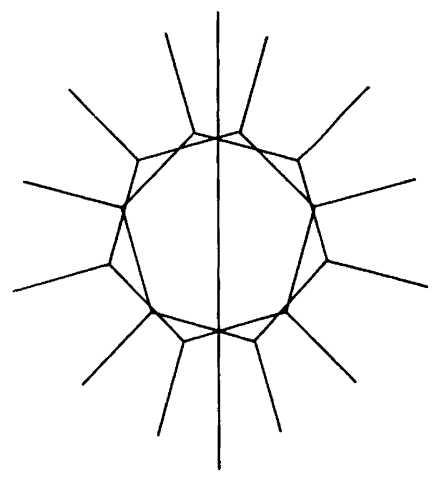

m

Fig. 1. The disorder of the HFB molecule.
It was rather difficult to find a weighting scheme which gave approximately the same mean value of $\omega \Delta F^{2}$ for different categories of reflections. The best results were obtained when $w=\sin ^{2} \theta / \lambda^{2}$ was used. The final $R$ value was 0.058 and $R_{\mathrm{w}}=\left[\Sigma w\left(F_{\mathrm{o}}-F_{\mathrm{c}}\right)^{2} / \Sigma w F^{2}\right]^{1 / 2}=0.073$. The maximum electron density in the final difference map is 0.10 $\mathrm{e} / \AA^{3}$. Lists of observed and calculated structure factors and anisotropic temperature factors may be obtained from the author on request. The final positional parameters and $U_{\text {eq }} / U_{\text {iso }}$ values are given in Table 1 . Bond distances, uncorrected and corrected for librational motion, ${ }^{11}$ and bond angles are given in Table 2 . The molecular packing is shown in Fig. 2.

Scattering factors were taken from Ref. 12. All calculations were performed on a VAX 8600 computer at the University of Troms $\varnothing$. The computer programs used are included in the Oxford CRYSTALS package. ${ }^{13}$

Lattice-energy calculations. Using the computer program PCK $83^{14}$ the lattice energy was calculated from the expression

$E=\sum_{\mathrm{j} k}-A_{\mathrm{jk}} r_{\mathrm{jk}}^{-6}+B_{\mathrm{jk}} \exp \left(-C_{\mathrm{jk}} r_{\mathrm{jk}}\right)+q_{\mathrm{j}} q_{\mathrm{k}} r_{\mathrm{jk}}^{-1}$.

All intermolecular distances $r_{\mathrm{jk}}$ up to $9 \AA$ were included in the sum. The non-bonded potential parameters $A_{\mathrm{jk}}, B_{\mathrm{jk}}$ and $C_{\mathrm{jk}}$ were taken from Refs. 15 and 16.

As the calculations of such parameters for $\mathrm{H}$ have been based on a slight foreshortening of the bond distance, ${ }^{16}$ $\mathrm{C}-\mathrm{H}$ distances of $1.00 \AA$ were assumed in the energy calculations. The $\mathrm{H}$ positions of the DMA molecule were therefore slightly modified. For the TMPDA-HFB complex no $\mathrm{H}$ positions were found in the crystal-structure determination, and they were therefore calculated using the crystal structure of TMPDA as a model. ${ }^{17}$

Net atomic charges, $q$, of -0.174 and 0.174 e for the $F$ and $\mathrm{C}$ atoms, respectively, of the HFB molecule were taken from Ref. 15. For the aromatic amines the atomic charges were calculated by the AM1-method, ${ }^{18}$ using the computer program GAUSSIAN $86 .{ }^{19}$ For the non-H atoms the molecular geometries of the experimental structures of the complexes were used in these calculations. The $\mathrm{C}-\mathrm{H}$ distances were, however, extended to $1.08 \AA$. The net charges calculated for the atoms of the TMA molecule are given in Table 3 . For the atoms of the dimethylamino groups and the $\mathrm{H}$ atoms bonded directly to the benzene rings in the TMPDA and the DMA molecule, no charges deviate more than $0.01 \mathrm{e}$ from those of the corresponding atoms in the TMA molecule. For the ring $\mathrm{C}$ atoms bonded to $\mathrm{N}$, the charge is 0.073 and $0.127 \mathrm{e}$ for TMPDA and DMA, respectively, and for the other atoms in the benzene rings the values are $-0.207 \mathrm{e}$ for TMPDA and -0.260 , -0.147 and -0.245 e for DMA in the $o$-, $m$ - and $p$-positions, respectively.

Energy calculations were performed for the molecular packing of the experimental structures and for the packing giving the lowest energy when the cell dimensions and the 
DAHL

Table 1. Positional parameters and equivalent or isotropic temperature factors $\left(\AA^{2}\right) .^{a}$

\begin{tabular}{|c|c|c|c|c|}
\hline Atom & $x$ & $y$ & $z$ & $U_{\mathrm{eq}}{ }^{b}$ or $U_{\text {iso }}$ \\
\hline$F(1)$ & $0.0834(6)$ & $0.2954(7)$ & $-0.1959(8)$ & 0.200 \\
\hline$F(2)$ & $0.1632(8)$ & $0.4300(7)$ & $0.0340(16)$ & $0.242(9)$ \\
\hline$F(3)$ & $0.2478(7)$ & $0.3778(7)$ & $0.3419(12)$ & $0.214(8)$ \\
\hline$F(4)$ & $0.2546(6)$ & $0.1921(8)$ & $0.4248(8)$ & $0.204(8)$ \\
\hline$F(5)$ & $0.1762(8)$ & $0.0587(6)$ & $0.1959(15)$ & $0.221(7)$ \\
\hline$F(6)$ & $0.0920(7)$ & $0.1103(8)$ & $-0.1103(11)$ & $0.228(7)$ \\
\hline$C(1)$ & $0.1265(7)$ & $0.2707(5)$ & $-0.0421(7)$ & 0.113 \\
\hline$C(2)$ & $0.1659(7)$ & $0.3385(6)$ & $0.0729(10)$ & $0.128(7)$ \\
\hline$C(3)$ & $0.2090(7)$ & $0.3120(6)$ & $0.2307(8)$ & $0.108(6)$ \\
\hline$C(4)$ & $0.2129(7)$ & $0.2177(5)$ & $0.2727(7)$ & 0.112 \\
\hline$C(5)$ & $0.1732(7)$ & $0.1500(5)$ & $0.1574(9)$ & $0.113(6)$ \\
\hline$C(6)$ & $0.1301(7)$ & $0.1764(6)$ & $0.0003(8)$ & $0.120(6)$ \\
\hline$C(7)$ & $0.6470(7)$ & 0.2500 & $0.0567(6)$ & $0.075(2)$ \\
\hline$C(8)$ & $0.6752(5)$ & $0.3371(3)$ & $0.1433(4)$ & $0.079(2)$ \\
\hline$C(9)$ & $0.7288(5)$ & $0.3371(3)$ & $0.3083(4)$ & $0.084(2)$ \\
\hline$C(10)$ & $0.7550(8)$ & 0.2500 & $0.3895(6)$ & $0.085(2)$ \\
\hline$C(11)$ & $0.5685(10)$ & $0.3372(5)$ & $-0.1983(6)$ & $0.127(3)$ \\
\hline$C(12)$ & $0.7561(11)$ & $0.4313(4)$ & $0.3994(7)$ & $0.128(3)$ \\
\hline$N(1)$ & $0.5913(9)$ & 0.2500 & $-0.1100(6)$ & $0.109(3)$ \\
\hline$H(1)$ & $0.674(6)$ & $0.3991(17)$ & $0.084(5)$ & $0.119(14)$ \\
\hline$H(2)$ & $0.785(7)$ & 0.2500 & $0.510(1)$ & $0.088(15)$ \\
\hline$H(3)$ & $0.541(7)$ & $0.3215(15)$ & $-0.316(1)$ & $0.134(16)$ \\
\hline$H(4)$ & $0.695(4)$ & $0.3726(29)$ & $-0.176(8)$ & $0.220(34)$ \\
\hline $\mathrm{H}(5)$ & $0.469(7)$ & $0.3782(23)$ & $-0.162(6)$ & $0.183(28)$ \\
\hline$H(6)$ & $0.820(8)$ & $0.4788(17)$ & $0.339(4)$ & $0.161(22)$ \\
\hline$H(7)$ & $0.838(8)$ & $0.4201(16)$ & $0.510(3)$ & $0.183(25)$ \\
\hline$H(8)$ & $0.626(2)$ & $0.4582(38)$ & $0.404(11)$ & $0.274(47)$ \\
\hline
\end{tabular}

aStandard deviations in parentheses. Occupancy factors for $F(1-6)$ and $C(1-6)$ are 0.5 .

${ }^{b} U_{\mathrm{eq}}=1 / 3 \sum_{\mathrm{i}} \sum_{\mathrm{j}} U_{\mathrm{ij}} a_{\mathrm{i}}^{*} a_{\mathrm{j}}^{*} a_{\mathrm{i}} a_{\mathrm{j}} \cos \alpha_{\mathrm{ij}}$

Table 2. Bond distances $(\AA)$, uncorrected and corrected for librational motion, and bond angles $\left({ }^{\circ}\right)$ for non-hydrogen atoms. ${ }^{a}$

\begin{tabular}{|c|c|c|c|c|}
\hline \multirow[t]{2}{*}{ Bond } & \multicolumn{2}{|l|}{ Distance/Å } & \multirow[t]{2}{*}{ Angle } & \\
\hline & Uncorrected & Corrected & & \\
\hline$F(1)-C(1)$ & $1.322(7)$ & 1.357 & $F(1)-C(1)-C(2)$ & $120.3(4)$ \\
\hline$F(2)-C(2)$ & $1.313(7)$ & 1.353 & $C(6)-C(1)-C(2)$ & $119.7(3)$ \\
\hline$F(3)-C(3)$ & $1.301(7)$ & 1.339 & $F(2)-C(2)-C(3)$ & $119.9(4)$ \\
\hline$F(4)-C(4)$ & $1.312(7)$ & 1.347 & $C(1)-C(2)-C(3)$ & $119.6(3)$ \\
\hline$F(5)-C(5)$ & $1.310(8)$ & 1.351 & $F(3)-C(3)-C(4)$ & $119.9(4)$ \\
\hline$F(6)-C(6)$ & $1.301(7)$ & 1.339 & $C(2)-C(3)-C(4)$ & $120.6(3)$ \\
\hline$C(1)-C(2)$ & $1.343(7)$ & 1.382 & $F(4)-C(4)-C(5)$ & $119.8(4)$ \\
\hline$C(2)-C(3)$ & $1.361(7)$ & 1.398 & $C(3)-C(4)-C(5)$ & $119.7(4)$ \\
\hline$C(3)-C(4)$ & $1.358(7)$ & 1.400 & $F(5)-C(5)-C(6)$ & $119.8(4)$ \\
\hline$C(4)-C(5)$ & $1.343(7)$ & 1.383 & $C(4)-C(5)-C(6)$ & $119.8(3)$ \\
\hline$C(5)-C(6)$ & $1.355(7)$ & 1.391 & $F(6)-C(6)-C(1)$ & $120.1(4)$ \\
\hline$C(6)-C(1)$ & $1.359(7)$ & 1.401 & $C(5)-C(6)-C(1)$ & $120.7(3)$ \\
\hline$C(7)-C(8)$ & $1.409(4)$ & 1.422 & $C(8)-C(7)-C(8)^{\prime}$ & $118.5(4)$ \\
\hline$C(8)-C(9)$ & $1.371(5)$ & 1.381 & $C(8)-C(7)-N(1)$ & $120.8(2)$ \\
\hline$C(9)-C(10)$ & $1.386(5)$ & 1.400 & $C(7)-C(8)-C(9)$ & $120.8(4)$ \\
\hline$C(9)-C(12)$ & $1.513(6)$ & 1.528 & $C(8)-C(9)-C(10)$ & $119.2(4)$ \\
\hline$C(7)-N(1)$ & $1.386(7)$ & 1.396 & $C(8)-C(9)-C(12)$ & $120.0(4)$ \\
\hline$N(1)-C(11)$ & $1.417(6)$ & 1.430 & $C(9)-C(10)-C(9)^{\prime}$ & $121.7(5)$ \\
\hline & & & $C(7)-N(1)-C(11)$ & $121.1(3)$ \\
\hline & & & $C(11)-N(1)-C(11)^{\prime}$ & $117.6(6)$ \\
\hline
\end{tabular}

Standard deviations in parentheses. All $\mathrm{C}-\mathrm{H}$ distances are $1.00(1) \AA$, all $\mathrm{N}-\mathrm{C}(11)-\mathrm{H}$ and $\mathrm{C}-\mathrm{C}(12)-\mathrm{H}$ angles are $109(1)^{\circ}$, the angles $\mathrm{H}-\mathrm{C}(\mathrm{Me})-\mathrm{H}$ are $106-116^{\circ}$, and the angles $\mathrm{C}-\mathrm{C}-\mathrm{H}(1)$ and $\mathrm{C}-\mathrm{C}-\mathrm{H}(2)$ are $119.1-120.8^{\circ}$. Symmetry operator: $x, 0.5-y, z$. 


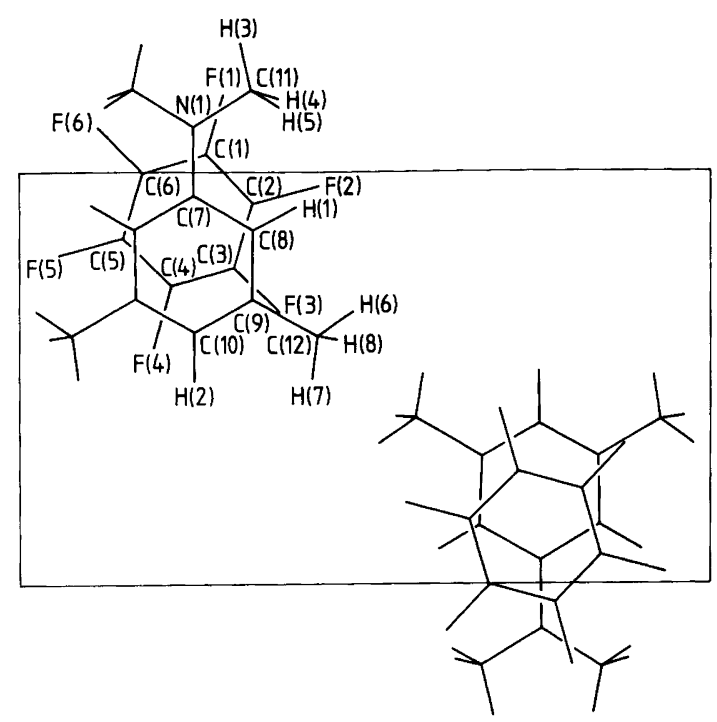

Fig. 2. The molecular packing viewed along [100]. Only one of the equivalent orientations of the HFB molecule is shown.

molecular positions and orientations were varied. The calculated packing should ideally be compared with the lowtemperature experimental structure. If it is compared with the room-temperature structure the difference in calculated energy is largely a result of the difference in packing density. In order to estimate the importance of the molecular positions and orientations, calculations were also made of the lowest energy after variation the cell dimensions only. Table 4 gives the calculated energy and the interplanar distance in the stack for the experimental structures and for the packings arrived at in these calculations. Fig. 3 shows the molecular overlap found experimentally and that giving the lowest calculated energy.

In all these calculations the Coulombic energy was found to be between 27.6 and $32.3 \%$ of the total energy. Moderate variations in the net atomic charges have some effect on the interplanar distance, but little effect on the molecular overlap in the packing giving the lowest calculated energy.

Table 3. Net atomic charges of the TMA molecule.

\begin{tabular}{lrll}
\hline Atom & $q$ & Atom & $q$ \\
\hline $\mathrm{C}(7)$ & 0.142 & $H(1)$ & 0.189 \\
$\mathrm{C}(8)$ & -0.261 & $H(2)$ & 0.185 \\
$\mathrm{C}(9)$ & -0.048 & $H(3)$ & 0.132 \\
$\mathrm{C}(10)$ & -0.236 & $H(4)$ & 0.105 \\
$\mathrm{C}(11)$ & -0.167 & $H(5)$ & 0.131 \\
$\mathrm{C}(12)$ & -0.310 & $H(6)$ & 0.128 \\
$\mathrm{~N}(1)$ & -0.429 & $H(7)$ & 0.135 \\
& & $H(8)$ & 0.135 \\
\hline
\end{tabular}

Table 4. Lattice energy $\left(\mathrm{kJ} \mathrm{mol}^{-1}\right)$ and mean interplanar distance $(\AA)$ for the experimental structure (first line), for the packing giving the lowest calculated energy after varying the cell dimensions only (second line), and after varying both cell dimensions and molecular positions and orientations (third line). For the experimental disordered structure of the TMPDA-HFB complex the calculations are based on the major orientation of the HFB molecule.

\begin{tabular}{llll}
\hline Compound & $\begin{array}{l}\text { Tempera- } \\
\text { ture/K }\end{array}$ & Energy & Distance \\
\hline TMPDA-HFB & 298 & -188.7 & 3.44 \\
& & -192.3 & 3.35 \\
TMA-HFB & 298 & -198.1 & 3.37 \\
& & -187.7 & 3.51 \\
DMA-HFB & \multirow{2}{*}{150} & -194.7 & 3.43 \\
& & -197.4 & 3.43 \\
& & -181.1 & 3.39 \\
& & -181.1 & 3.40 \\
& & -182.1 & 3.39 \\
\hline
\end{tabular}

\section{Discussion}

The large thermal parameters of the HFB molecule are largely the result of librational motion. The r.m.s. angles of libration are $11.9,8.1$ and $5.9^{\circ}$ about the principal librational axes, with the largest axis being nearly perpendicular to the molecular plane. These values are approximately the same as those observed for the complex with $N, N$-di-

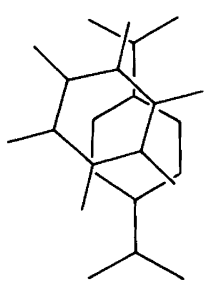

(a)

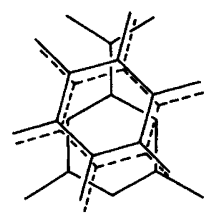

(c)

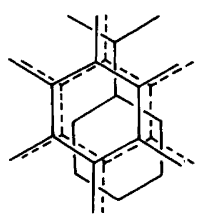

(e)

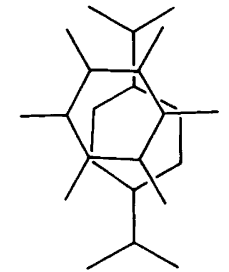

(b)

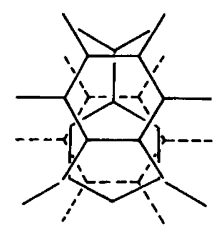

(d)

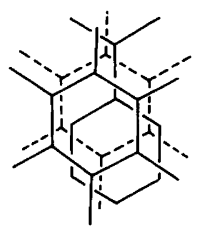

(f)
Fig. 3. The overlap of the molecules viewed perpendicular to their average plane for the experimental structure and for the packing giving the lowest calculated energy for the HFB complex with TMPDA [(a) and (b), respectively], TMA [(c) and (d)] and DMA [(e) and (f)]. Only the major orientation of the HFB molecule is shown in (a). 
methyl-p-toluidine, but larger than those observed for the complex with TMPDA and with DMA at room temperature. $^{20}$

Bond angles and corrected bond distances are approximately as expected. A relatively short intermolecular distance in the stack of $3.486(6) \AA$ between $\mathrm{C}(5)$ and $\mathrm{C}(8)$ $\left(-1+x, \frac{1}{2}-y, z\right)$ and one of $2.65(3) \AA$ between $\mathrm{F}(4)$ and $\mathbf{H}(3)\left(x, \frac{1}{2}-y, 1+z\right)$, which belong to different stacks, were observed.

No non- $\mathrm{H}$ atoms of the TMA molecule deviate significantly from a least-squares plane through the molecule, except $\mathrm{C}(11)$ which is $0.032(8) \AA$ out of this plane. The approximately planar geometry of the $\mathrm{N}$ atoms indicates that TMA is a $\pi$-donor rather than an $n$-donor in this complex. The angle between the planar part of this molecule and the HFB molecule, which is almost exactly planar as a result of the constraint, is $3.1^{\circ}$. The interplanar distances, defined as the separations of the ring centres projected onto the mean plane normal, are 3.498(4) and $3.530(4) \AA$ on the two sides of each molecule.

The mean interplanar distance giving the lowest calculated energy is shorter than that observed both in the TMA-HFB complex and in the TMPDA-HFB complex (Table 4). However, at room temperature the interplanar distance in the DMA-HFB complex is $3.52 \AA$. If the temperature dependence is the same as for the DMA-HFB complex, the interplanar distances at low temperatures in the two other complexes are shorter than those giving the lowest calculated energies.

In the structure of the TMA-HFB complex the molecular overlap is approximately the same on both sides of each molecule (Fig. 3). The benzene rings of the partner molecules are twisted $15^{\circ}$ relative to each other. This twisting is approximately the same as that observed in the TMPDA-HFB complex and is not consistent with the pattern observed for other HFB complexes. In the packing giving the lowest calculated energy the rings are twisted $30^{\circ}$ relative to each other and the overlap is different on the two sides. Substantial differences between the overlap in the experimental structure and that giving the lowest calculated energy are observed also for the TMPDA-HFB complex. For the DMA-HFB complex, however, these differences are extremely small.

For the interpretation of these results it should be remembered that the barrier to rotation of the HFB molecule in these complexes is very low. For the TMA-HFB complex the difference is small between the lowest energy calculated with and without variation of the molecular orientations and positions (Table 4, lines 3 and 2). This difference may well be compensated for by a possible entropy effect. The results seem, however, to indicate that interactions not included in the expression used for the non- bonded potentials have some effect on the structure of the TMPDA-HFB complex and possibly also on that of the TMA-HFB complex. The ionization potentials of TMPDA and TMA, 6.25 and $6.93 \mathrm{eV}$, respectively, are lower than that of DMA, $7.12 \mathrm{eV}, 4.5$ and the charge-transfer interactions with HFB are thus expected to be stronger. It is thus possible that the observations are the result of such interactions.

Acknowledgements. The author thanks Lars K. Hansen for help in the use of the diffractometer and Leif Sæthre for help with the AM1 calculations.

\section{References}

1. Dahl, T. Acta Chem. Scand., Ser. A 42 (1988) 1.

2. Dahl, T. Acta Chem. Scand., Ser. A 33 (1979) 665.

3. Beaumont, T. G. and Davis, K. M. C. J. Chem. Soc. B (1967) 1131.

4. Lias, S. G., Jackson, J. A., Argentar, H. and Liebman, J. F. J. Org. Chem. 50 (1985) 333.

5. Laidlaw, R. K., Miura, Y., Grant, J. L., Cooray, L., Clark, M., Kispert, L. D. and Metzger, R. M. J. Chem. Phys. 87 (1987) 4967.

6. Dahl, T. Acta Crystallogr., Sect. C 41 (1985) 931.

7. Dahl, T. Acta Crystallogr., Sect. B 37 (1981) 98.

8. North, A. C. T., Phillips, D. C. and Mathews, F. S. Acta Crystallogr., Sect. A 24 (1968) 351.

9. Larson, A. C. In: Hall, S. R., Ed., Crystallographic Computing, Munksgaard, Copenhagen 1970, p. 91.

10. Waser, J. Acta Crystallogr. 16 (1963) 1091.

11. Schomaker, V. and Trueblood, K. N. Acta Crystallogr., Sect. B 24 (1968) 63.

12. International Tables for $X$-Ray Crystallography, Kynoch Press, Birmingham 1974, Vol. 4, p. 99.

13. Watkins, D. J. and Carruthers, J. R. CRYSTALS: User Manual, Chemical Crystallography Laboratory, University of Oxford, Oxford 1983.

14. Williams, D. E. PCK83: A Crystal Molecular Packing Analysis Program, University of Louisville 1983; Quantum Chemistry Program Exchange, Program No. 481, Department of Chemistry, Indiana University, Indiana.

15. Williams, D. E. and Houpt, D. J. Acta Crystallogr., Sect. B 42 (1986) 286.

16. Williams, D. E. and Cox, S. R. Acta Crystallogr., Sect. B40 (1984) 404.

17. Ikemoto, I., Katagiri, G., Nishimura, S., Yakushi, K. and Kuroda, H. Acta Crystallogr., Sect. B 35 (1979) 2264.

18. Dewar, M. J. S., Zoebish, E. G., Healy, E. F. and Stewart, J. J. P. J. Am. Chem. Soc. 107 (1985) 3902.

19. Frisch, M. J., Binkley, J. S., Schlegel, H. B., Raghaavachari, K., Melius, C. F., Martin, L., Stewart, J. J. P., Bobrowicz, F. W., Rohlfing, C. M., Kahn, L. R., de Frees, D. J., Seeger, R. A., Whiteside, R. A., Fox, D. J., Fleuder, E. M. and Pople, J. A. GAUSSIAN 86: Carnegie-Mellon Quantum Chemistry Publishing Unit, Pittsburg PA 1984.

20. Dahl, T. Acta Crystallogr., Sect. B 33 (1977) 3021.

Received June 27, 1988. 